\title{
The Profiling of International Roughness Index (IRI) Based on Lagrangian Method
}

\author{
Schun T. Uechi ${ }^{1}$, Hiroshi Uechi ${ }^{2}$ \\ ${ }^{1} 902$ S Citron, Anaheim, CA, USA \\ ${ }^{2}$ Osaka Gakuin University, Suita, Osaka, Japan \\ Email: quantum2006hara@gmail.com, uechi@ogu.ac.jp
}

How to cite this paper: Uechi, S.T. and Uechi, H. (2018) The Profiling of International Roughness Index (IRI) Based on Lagrangian Method. World Journal of Engineering and Technology, 6, 885-902. https://doi.org/10.4236/wjet.2018.64059

Received: October 11, 2018

Accepted: November 20, 2018

Published: November 23, 2018

Copyright (๑) 2018 by authors and Scientific Research Publishing Inc. This work is licensed under the Creative Commons Attribution International License (CC BY 4.0).

http://creativecommons.org/licenses/by/4.0/

\begin{abstract}
The phenomenological equations of motion for the international roughness index (IRI) have been reviewed and discussed in terms of Lagrangian method in physics. The current paper proposes a practical, two-dimensional model for studying essentially three-dimensional, vibrating, and mechanical systems (vehicles). The purpose is to provide a new profiling method for IRI, which is practical in computations and compatible with traditional profiling for roughness of a road-surface. The modern technology employs elaborated sensors such as gyro sensor, Global Positioning System (GPS), magnetometer sensor, and accelerometer to measure high-speed longitudinal motions, resulting in time series of big-data expressed as compressed longitudinal spikes. The time series of longitudinal spikes obtained from high-speed longitudinal motions are traditionally considered as a background noise for constructing a profile. The conventional IRI is calculated from big-data of the road profile by employing statistical method, but the Lagrangian model dynamically determines the road profile. The useful concept and relation among the road-roughness function $z(t)$, associated roughness index (ARI), acceleration and position are introduced and examined in the present paper. The associated roughness index (ARI) defined by the current dynamical approach is examined by applying virtual simulations which represent roughness of a road-surface. The current theoretical model supports and compensates information of interpreting a profile of IRI and elucidates physical meanings for the roughness index of a road-surface.
\end{abstract}

\section{Keywords}

International Roughness Index (IRI), Associated Roughness Index (ARI), Virtual Simulations of Roughness of a Road-Surface 


\section{Introduction}

The International Roughness Index (IRI) is the standard roughness index of pavement management employed to acquire quantitative longitudinal surface-bump data of road surfaces. It is a calibration of a road roughness measurement by using a quarter-car vehicle math model, with units of the road roughness index (in/mi, $\mathrm{m} / \mathrm{km})$, commonly used worldwide [1]. In the early 1960s, General Motors Research (GMR) developed a Profilometer capable of measuring dynamic portion of a road profile which is responsible for inducing vehicle ride motions [2]. The IRI, since introduced in 1986, has been used worldwide for evaluating and managing road systems, maintenance and repair of roadways in order to optimize pavement conditions, and the work was continued by The World Bank and others [3]. The measurement of IRI is required for data provided to the United States Federal Highway Administration, and American Society for Testing and Materials (ASTM International) administrates standard practice for computing IRI of roads from longitudinal profile measurements.

The calibration requires comparing measured data statistically determined in several different ways with measured road-surface values. In other words, true longitudinal changes of road-surface are measured as characteristic changes or patterns depending on measuring instruments and statistical assumptions. Therefore, it is necessary to check mathematical and physical definitions of true longitudinal changes and patterns for calibration references. Although some mathematical linear and nonlinear models are introduced, they are not transparent in terms of physics, and so, we introduce a physical model based on Lagrangian approach in the Section 2.

The road roughness and surface-bump measurements have been applied primarily for restoring and repairing roads which are undermined by longtime use of roads or earthquakes. Hence, the measurement technology of IRI has become more important as modern technology advances with elaborated sensors such as gyro sensor, Global Positioning System (GPS), and magnetometer sensor, etc. It could be applied to space missions, such as the moon and Mars' explorations as unmanned surface vehicles (USV), or wave energy converter (WEC), because theory and technology related to IRI are essentially related to dynamical motions of a three-dimensional system affected by internal oscillations and trembles, heat and energy dissipations, external waves and impacts.

Mathematical models of vehicle's response have been investigated since the 1940s for evaluations of airplanes and military vehicles, and road-roughness profiles with conventional survey methods, and converting them into a compatible profile with statistical methods for big-data seemed strenuous to achieve by considering suspension systems of a vehicle in those old days. However, the experiment of conducting mechanical responses to external impacts was essential for applications to an aircraft failure moving a runway, a military vehicle traversing rough geological areas, the standard fabrication for 
steel affecting fast-growing railroad, automobile industries, etc. These practical necessities essentially required technical standards for a wide range of materials, products and transportation systems.

The roughness measure index from an ideal response-type road roughness measurement system (RTRRMS) is obtained using a quarter-car simulation (QCS) [1] [2] [3]. The reference is used to choose model parameters to offer maximum correlation with existing RTRRMSs. The way a tire envelops small bumps was found to have a critical influence when the QCS was used at low speed, and the tire enveloping effect was studied for the model when low-speed simulations were performed. Though the measurement of IRI seems direct and simple experiments, a three-dimensional vibrational system with heat dissipation, interacting with external environment is extremely complicated. This is the reason why Lagrangian approach to IRI is employed to study a profiling of IRI.

The Golden Car simulation from the project of National Cooperative Highway Research Program (NCHRP) [4] in USA was one of references under the condition that a standard simulation speed would be needed for the IRI. After processing International Road Roughness Experiment (IRRE) data, correlations between a road profile index and the response-type systems were reported with two vehicle simulations based on the Golden Car parameters: a quarter-car and a half-car. The quarter-car was selected for the IRI, and the consensus of researchers was that the standard speed should be $80 \mathrm{~km} / \mathrm{h}$ (49.7 mph) [5].

However, it is commonly known that modern vehicle's effectiveness in insulating drivers and passengers from vibrations, bumps or corrugations in the road surface has well advanced, which has been studied as ride quality. The ride quality is related to suspension components which connect a vehicle to wheels, such as springs, shock absorbers and bushings. Hence, a calibration of roughness index of a road-surface depends on ride quality. One can use a two-wheeled car, Segway, with accelerometer and GPS to obtain data for IRI. In other words, instruments to measure a profile of road-surface have their own characteristic properties to respond longitudinal speed or acceleration caused by impacts of a road-surface. A road profile corresponding to impacts, bumps and slopes will be studied in detail by the current theoretical model.

Applications of gyroscopes include inertial navigation systems, such as in the Hubble telescope, ships, aircraft and spacecraft, vehicles etc, and Global Positioning System (GPS) is a device that is capable of receiving information from GPS satellites and calculating the device's geographical position [6]. In principle, by determining an object's acceleration from accelerometer of smartphone and position data from GPS, it could be possible to determine a profile of roughness of a road-surface. The vibrations of vehicles, dissipation phenomena induced by mechanical or hydraulic devices, damping and absorptions of shock impulses make direct connections between mechanical motions and statistical data extremely difficult.

The model based on Lagrangian method can apply for any vehicle and gravity in general which have accelerometer and GPS. The road flatness assumption [7] 
is not necessary in the center of mass analysis. In other words, if the deviation of longitudinal position from the center of mass is zero or smooth oscillation-damping to zero, the horizontal surface or flat surface can be perceived parallel to local reference frame. The center of mass analysis of two-dimensional coupled spring system, model Lagrangian and equations of motion from centers of gravity are discussed in Section 2.

The main purpose of this paper is to closely examine correlations between the data of IRI and longitudinal surface-bump data obtained by dynamical method, and explain mechanism in terms of physics by employing Lagrangian dynamical approach [8] [9] [10] [11] [12]. The concepts such as the profile of a road-surface, associated roughness index (ARI), acceleration and position are introduced, which could assist in deriving a consistent road profile from IRI. They are explicitly exhibited in Section 3. Conclusions are in Section 4.

\section{Model Lagrangian}

The coupled sprung and unsprung system is mechanically perspicuous to understand, but it is a complicated vibrational and dissipative system when suspensions and weight distributions, impact forces exerted from a rough road-surface are simultaneously considered. Mechanical vibrations in longitudinal directions are mainly considered, and tires and body of the side shown in Figure 1 move identically as those of the other side (no strains and deformations). These assumptions are the two-dimensional simplification of complex three-dimensional motions and reduce independent variables significantly. The two-dimensional simplification is used for the model Lagrangian, but characteristic properties of realistic motions and vibrations for constructing IRI can be specifically obtained, which will be shown by computer simulations.

Based on simplifications shown in Figure 1, the model Lagrangian, $L$, for a system of coupled masses to investigate longitudinal surface-bump of road is given by:

$L=\frac{1}{2} m_{1} \dot{x}^{2}+\frac{1}{2} m_{2} \dot{y}^{2}-\frac{1}{2} k_{1}\left\{\left(x_{0}-x\right)-\left(y_{0}-y\right)\right\}^{2}-\frac{1}{2} k_{2}\left(y_{0}-y\right)^{2}-m_{1} g x-m_{2} g y$,

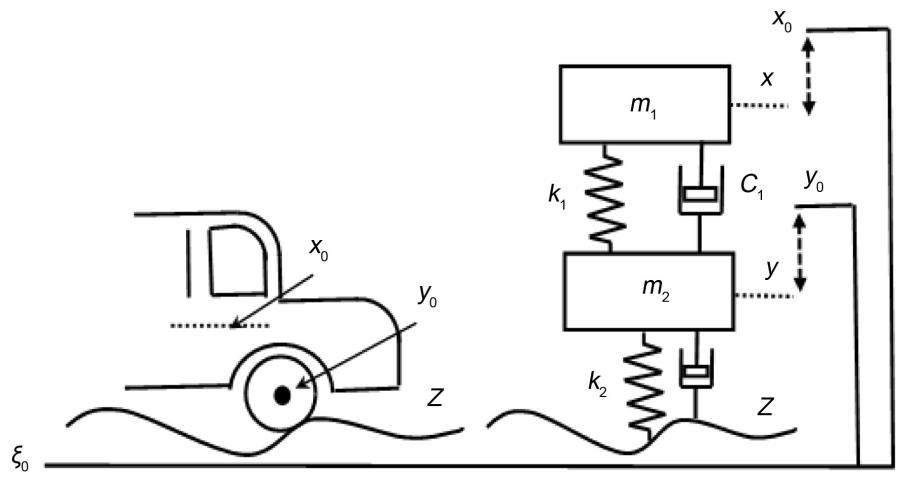

Figure 1. The simplified two-dimensional image of a sprung and unsprung system. 
where the notation of differentiation with respect to time, $\mathrm{d} x / \mathrm{d} t=\dot{x}$ and $\mathrm{d}^{2} x / \mathrm{d} t^{2}=\ddot{x}$, is used. The spring constants, $k_{1}$ and $k_{2}$, and masses, $m_{1}$ and $m_{2}$, are shown in Figure 1. The natural lengths of springs are respectively $x_{0}$ and $y_{0}$, which are hypothetically measured when the gravity is switched off, $g=0$. Then, the vertical positions of masses, $x(t)$ and $y(t)$, are considered to be defined when the gravity, $g$, is switched on. The natural lengths, $x_{0}$ and $y_{0}$, will be changed to variables of centers of masses of the car and tire, $x_{G}$ and $y_{G}$, respectively. Then, physical variations of positions will be measured separately from the center of masses of the car and tire as $\Delta x(t)=x(t)-x_{G}$ and $\Delta y(t)=y(t)-y_{G}$. Hence, the flatness of road surface is defined by $\Delta x(t)=0$, resulting in $x(t)=x_{G}$ or a damping oscillation of $\Delta x(t)$ around $x_{G}$. The profile of roughness of road-surface, bumps and corrugations and up-down slopes can be distinguished based on the flatness of road-surface.

The gravitational constant, $g=9.8 \mathrm{~m} / \mathrm{s}^{2}$, changes according to geologic structure of environment, low or high latitudes and altitudes, on the surface of moon, or Mars, etc.; however, in the center of mass system, the gravitational constant $g$ does not appear in the equation of motion, indicating that the current analysis can be applied in general circumstances.

Because of dissipative mechanism, friction and heat generation of threedimensional machines, it is necessary in practice to introduce the power function $P$ in order to compensate for dissipative phenomena in mechanical systems [9], written as,

$$
P=\frac{1}{2}\left(C_{1} \dot{x}^{2}+C_{2} \dot{y}^{2}\right)
$$

The parameters, $C_{1}, C_{2}$, are phenomenological constants, which will be optimally adjusted in data simulations. In reality, these constants may correspond to effects of heat-generation, duration of trembles produced by a complicated three-dimensional mechanical system, shock absorbers used for mechanical or hydraulic devices designed to damp and absorb shock impulses. The kinetic energy of shock-impulse is converted into heat, and the dissipation of heat is commonly experienced after driving a vehicle in everyday life. The shock absorbers are expressed in the form of a dash-pot depicted in Figure 1 with $C_{1}$ and $C_{2}$.

The equation of motion defined by Euler-Lagrange equation and power function is given by:

$$
\frac{\mathrm{d}}{\mathrm{d} t} \frac{\partial L}{\partial \dot{q}}-\frac{\partial L}{\partial q}=-\frac{\partial P}{\partial \dot{q}},
$$

The power function, $P$, has the dimension of power (joule/s), representing phenomenological mechanism of dissipation. One has to include the effect of roughness of road $z(t)$ shown in Figure 1. Because $z(t)$ is independent of internal energy and energy-dissipation mechanism, it is considered as an external force with the spring constant $k_{2}$ as $-k_{2}(y(t)-z(t))$. The function $z(t)$ is directly related to the roughness of a road-surface and used to interpret 
IRI data.

Based on discussions above, the coupled differential equations for sprung and unsprung masses are given by:

$$
\begin{gathered}
m_{1} \ddot{x}+C_{1} \dot{x}-k_{1}\left\{\left(x_{0}-x\right)-\left(y_{0}-y\right)\right\}+m_{1} g=0, \\
m_{2} \ddot{y}+C_{2} \dot{y}+k_{1}\left\{\left(x_{0}-x\right)-\left(y_{0}-y\right)\right\}-k_{2}\left(y_{0}-y\right)+m_{2} g=-k_{2}(y-z(t)),
\end{gathered}
$$

and initial conditions are, $x(0)=x_{0}, y(0)=y_{0}$, and $\dot{x}(0)=v_{x}, \dot{y}(0)=v_{y}$. The initial conditions are defined from inertial frame $\xi_{0}$ as shown in Figure 1. Rigorously speaking, it is difficult to specify the inertial coordinate frame, because the Earth is not an inertial system. However, as we know from our experience, motions of masses are conveniently discerned and studied by tracing centers of masses. The centers of masses for $m_{1}$ and $m_{2}$ are respectively denoted as $x_{G}$ and $y_{G}$ and used for physical and mathematical analyses to construct profiles of IRI. The motions around centers of masses are practical and useful for Roughness Index measurement as well as human sense of recognition.

The centers of masses are defined as $x(t)-x_{G}=0$ and $y(t)-y_{G}=0$ at $t \rightarrow \infty$, and so, we have the following equations,

$$
\begin{aligned}
& -k_{1}\left\{\left(x_{0}-x_{G}\right)-\left(y_{0}-y_{G}\right)\right\}+m_{1} g=0, \\
& k_{1}\left\{\left(x_{0}-x_{G}\right)-\left(y_{0}-y_{G}\right)\right\}-k_{2}\left(y_{0}-y_{G}\right)+m_{2} g=0,
\end{aligned}
$$

leading to:

$$
\begin{aligned}
& x_{0}=\frac{\left(m_{1}+m_{2}\right) g}{k_{2}}+\frac{m_{1} g}{k_{1}}+x_{G}, \\
& y_{0}=\frac{\left(m_{1}+m_{2}\right) g}{k_{2}}+y_{G} .
\end{aligned}
$$

Hence, equations of motion (2.4) and (2.5) can be practically defined with $x_{G}$ and $y_{G}$ as:

$$
\begin{gathered}
m_{1} \ddot{x}+C_{1} \dot{x}-k_{1}\left\{\left(x_{G}-x\right)-\left(y_{G}-y\right)\right\}=0 \\
m_{2} \ddot{y}+C_{1} \dot{y}+k_{1}\left\{\left(x_{G}-x\right)-\left(y_{G}-y\right)\right\}-k_{2}\left(y_{G}-y\right)=-k_{2}(y-z(t)) .
\end{gathered}
$$

The values of masses, $m_{1}, m_{2}$, and centers of masses, $x_{G}, y_{G}$ can be empirically fixed. The variations, $\Delta x(t)=x(t)-x_{G}$ and $\Delta y(t)=y(t)-y_{G}$, are vertical displacements from centers of masses, representing reactions to roughness of a road surface. The variations $\Delta x(t)$ and $\Delta y(t)$ will be shown in detail by solving Equations (2.8) and (2.9). Spring constants, $k_{1}, k_{2}$ and phenomenological constants, $C_{1}, C_{2}$, are optimally adjusted by numerical simulations with the initial condition that the external force coming from the roughness of road surface is zero, $y(t)-z(t) \equiv 0$, resulting in $x(t)=x_{G}$ and $y(t)=y_{G}$.

One should note that a roughness of road-surface is proposed in the beginning by introducing the hypothetical, roughness-function $z(t)$ in the current Lagrangian method. Then, the profiles of associated roughness index 
(ARI), changes of $\Delta x(t)$ and acceleration $\Delta \alpha(t)$ are evaluated. On the other hand, IRI is statistically calculated from a road profile measured in different ways using the data of a Golden car (a quarter car or a half car) as briefly reported in introduction.

The parameters used by the Golden-Car assumption, for example, are reported as [13] [14],

$k_{1} / m_{1}=63.3\left[1 / \mathrm{s}^{2}\right], C_{1} / m_{1}=6.0[1 / \mathrm{s}], k_{2} / m_{1}=653.0\left[1 / \mathrm{s}^{2}\right], m_{2} / m_{1}=0.15$,

and we use MKS system of units (meter, kilogram, second) for measurement of physical quantities to avoid any confusion in the research area. The impact from $z(t)$ is supposed to affect $y(t)$ directly, whereas $y(t)$ is affected by $y(t)$, which would be assured by $m_{1}>m_{2}$. Though parameters depend on suspension and dissipative mechanism of a system, the profile of road-surface or patterns of impacts should be model-independently interpreted.

The road-surface profiles and patterns, small cracks or dents, bumps or corrugations would be simulated with $z(t)$ by employing the piecewise continuous step function:

$$
\theta(t-1)= \begin{cases}1 & t>1 \\ 0 & t<1\end{cases}
$$

The perfectly smooth road surface is represented as $z(t)=0$, which is parallel to the local inertial frame of reference $\xi_{0}$. Hence, the external driving-force, $-k_{2}(y-z(t))$, in the left-hand side of (2.9), appears only when the road surface deviates from the local horizontal surface parallel to the frame of reference, $\xi_{0}$.

The coupled differential Equations (2.8) and (2.9), can be completely decoupled by the differential operator method [15] as the 4th-order linear differential equations for $x(t)$ and $y(t)$, which can be integrated analytically. However, coefficients of the 4th-order differential equations result in algebraic equations, which is too complicated to pursue for practical calculations. Though the operator method can be used for numerical simulations even in case when parameters, $k_{1}, k_{2}$ and $C_{1}, C_{2}$ change in time, the 4th-order differential equations could become physically difficult to interpret. Our model is based on Lagrangian dynamics in physics and a phenomenological method for dissipative systems with external forces. Nonlinear couplings are not necessarily required, whose effects may be manifested by way of dissipative, vibrational mechanisms.

\section{Simulations of IRI}

The purpose of two-dimensional simulations of motion is to extract characteristic properties of complicated, real three-dimensional systems, which produces correlations between roughness of road-surface and IRI. The difference of physical meaning of roughness index deduced from statistical and dynamical method is discussed, and the profiles of the roughness index generated by model simulations are explicitly shown. 


\subsection{The Definition of IRI by the Conventional and Current Deterministic Model}

It is commonly said that big-data is so big and complex that traditional dataprocessing application software is inadequate. The speed at which the data is generated and processed is so fast that it is impossible to know how much and what kind of noise is in the data. Hence, the statistical approach is employed to define changes of acceleration and position from the big data, and samples are assumed independent and have identical probability distribution so that one can process raw data, assuming minimizations of error effects on signals.

The following procedure is the conventional statistical method to extract signals from the big data of IRI [16]. The acceleration is defined by dataprocessing of accelerometer and GPS data as:

$$
\boldsymbol{a}_{w}\left(t_{i}\right)=\boldsymbol{a}\left(t_{i}\right)-\frac{1}{N} \sum_{j=i-N}^{i+N} \boldsymbol{a}\left(t_{j}\right),
$$

where $N$ is the number of neighbors around time $t_{i}$, and one has to clearly specify the background noise distribution during the time from $i=0$ to $i=i_{\max }$. In this approach, it is imperative to show the reasonable convergence of computations which should be independent of choosing $N$. This would be difficult to achieve in numerical calculations because of unknown back-ground noise, three-dimensional vibrations and trembles of a system. In other words, since the time-calibration or recording-time $t_{i}$ in (3.1) using modern technology is so fast and different from that of human-scale time-recognition, the recording and processing time-scale of big-data is not practical for numerical calculations of an application software; it is not possible to distinguish real vibrations from background disturbances. These intrinsic problems are known as velocity and veracity (how much noise is in the data) in big data.

In the dynamical method, motions are precisely determined by equations of motion. For example, the Earth moves around the Sun according to the equation of motion, though it would fluctuates a little around its orbit, and hence, in terms of dynamics, big-data and statistical properties must be subjected to the orbit determined by equations of motion. The IRI data and position of $x(t)$ are calculated by integrating smoothed data of (3.1), but one should be careful that the acceleration $a(t)$ in (3.1) is a very rapidly oscillating function, which makes difficult to detect true values in a measured signal and obtain relations among accelerations, positions and road profiles, $z(t)$.

The time-dependent vibrational motions of centers of masses and tires, spring motions are all determined by equations of motion, but three-dimensional, structural vibrations and trembles are unknown and impacts of road-surface are external. The Lagrangian dynamical approach and big-data analysis have their own advantages in deterministic and statistical point of views, respectively. Therefore, the current dynamical model would help complement statistical calculations of the Equation (3.1) in order to construct IRI profiles.

Starting from acceleration data obtained by conventional statistical method 
(3.1), positions, $x(t)$ and $y(t)$, are obtained by integration, and the profile of IRI at arbitrary section is defined in continuous expression as [13]:

$$
\operatorname{IRI}_{t_{i}}=\frac{1}{L}\left|\int_{t_{0}}^{t_{i}} \mathrm{~d}(x(t)-y(t))\right|
$$

where $L$ is the horizontal distance along the local reference frame, $\xi_{0}$, on which IRI measurement from $t_{0}$ to $t_{i}$ is performed. Alternatively, the definition of IRI can be obtained as the solution to the difference equation in discrete form:

$$
\Delta\left(\operatorname{IRI}_{t}\right) \equiv \operatorname{IRI}(t+1)-\operatorname{IRI}(t)=\frac{1}{L}(x(t)-y(t))
$$

where the unit of time is defined by $t_{i+1}-t_{i}=\Delta t \equiv 1$ in (3.3). The formal solution to the difference equation is written by,

$$
\operatorname{IRI}_{t}=\frac{1}{L} \Delta^{-1}(x(t)-y(t))
$$

resulting in the solution of the summation form given by,

$$
\operatorname{IRI}_{t_{i}}=\frac{1}{L} \sum_{t_{j}=0}^{t_{i}-1}\left(x\left(t_{j}\right)-y\left(t_{j}\right)\right)
$$

Since the original definition of IRI is defined by the absolute value of (3.5), we finally obtain:

$$
\operatorname{IRI}_{t_{i}}-\operatorname{IRI}_{t_{i}=0}=\frac{1}{L}\left|\Delta x\left(t_{i}\right)-\Delta y\left(t_{i}\right)\right|,
$$

where the initial starting value $\operatorname{IRI}_{t_{i}=0}=0 ; \Delta x\left(t_{i}\right)=x(t)-x_{G}$ and $\Delta y\left(t_{i}\right)=y(t)-y_{G}$, because $x(0)=x_{G}$ and $y(0)=y_{G}$. The definition of IRI in (3.2) is equivalent to (3.6), only if (3.1) gives true values of acceleration.

If the speed of a measuring vehicle is constant given by $V_{0}(\mathrm{~m} / \mathrm{s})$ with a measurement time $t$, then the horizontal distance is $L=V_{0} t(\mathrm{~m})$. In the current model, the acceleration data (3.1) is obtained from the second derivative of the variation, $\Delta x(t)=x(t)-x_{G}$. By introducing road-surface functions $z(t)$, the associated roughness index (ARI), acceleration $\Delta \alpha(t)$, and position $\Delta x(t)$ will be shown in the following section.

\subsection{Model Simulations of IRI}

The response-type measuring systems for vertical $(x, y)$ data are assumed to be obtained from the Golden Car: a quarter-car and a half-car, slowly moving horizontally. The profiling of IRI data would be changed with the speed of the Golden Car, because information of the roughness of a road-surface will be lost as faster a vehicle' speed is set off. It may depend on how accurate data should be required for repairs or improvements, evaluation and management of road systems. The Australian Road Research Board (ARRB) collects transport data for a walking profiler, measured at walking speed [17]. The centers of masses are fixed as, $x_{G}=0.50(\mathrm{~m})$ and $y_{G}=0.30(\mathrm{~m})$, and model parameters are fixed in all simulations as:

$$
m_{1}=300(\mathrm{~kg}), m_{2}=0.25 m_{1}, k_{1}=8.0 m_{1}, k_{2}=m_{1}, C_{1}=6.0 m_{1}, C_{2}=0.05 m_{1} .
$$


The results and patterns of computer simulations are almost constant by changes of the mass $m_{1}$, whereas solutions sensitively depend on ratios of $m_{2} / m_{1}, k_{2} / k_{1}, C_{2} / C_{1}$. The simulations on the differential Equations (2.8) and (2.9) are executed by employing the following profile of road-surface, $z(t)$.

\subsection{1. $z(t)=$ A Rectangular Convex Impact: Figure 2(a)}

A rectangular convex impact is introduced for the simplest roughness of road-surface simulations by employing step functions as follows:

$$
z(t)=h_{1}\left(\theta\left(t_{1}\right)-\theta\left(t_{\text {endl }}\right)\right)
$$

and the time steps, $t_{1}$ and $t_{\text {end1 }}$, are starting and ending time of the rectangular impact, respectively. The height of a rectangular-impact, $h_{1}=0.01(\mathrm{~m})$, and time-width, $\Delta t_{w}=\left|t_{1}-t_{\text {end1 }}\right|=2$ (s) are shown in Figure 2(a). One should note that the width of a rectangular impact depends on the speed of a measuring vehicle such that $\Delta L_{w}=V_{0} \Delta t_{w}$, where $V_{0}$ is the horizontal speed of a measuring vehicle. The profile of a road-surface is assumed to be measured at walking speed in the current simulation. Because the profiling of a high-speed data requires a big-data analysis and computational time, the current model simulations should be virtually expanded when they are compared with those of IRI.

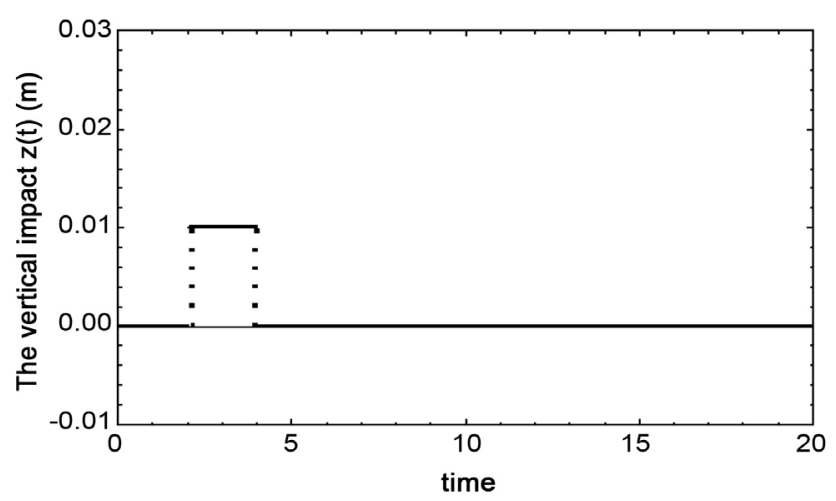

(a)

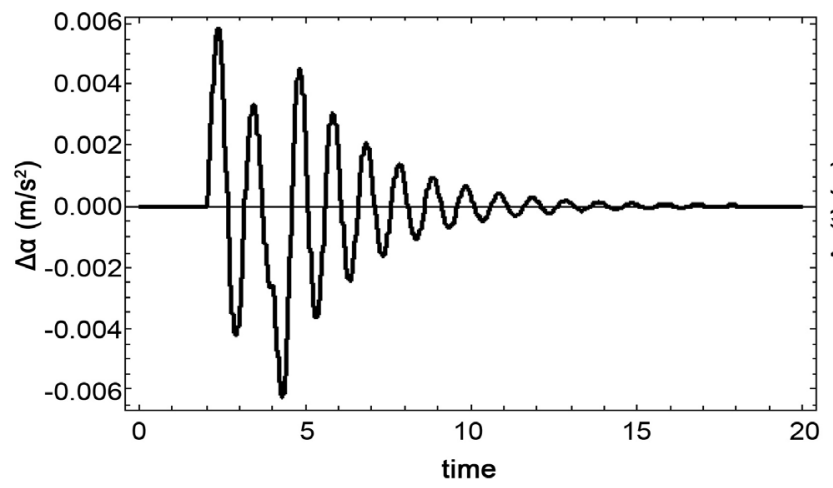

(c)

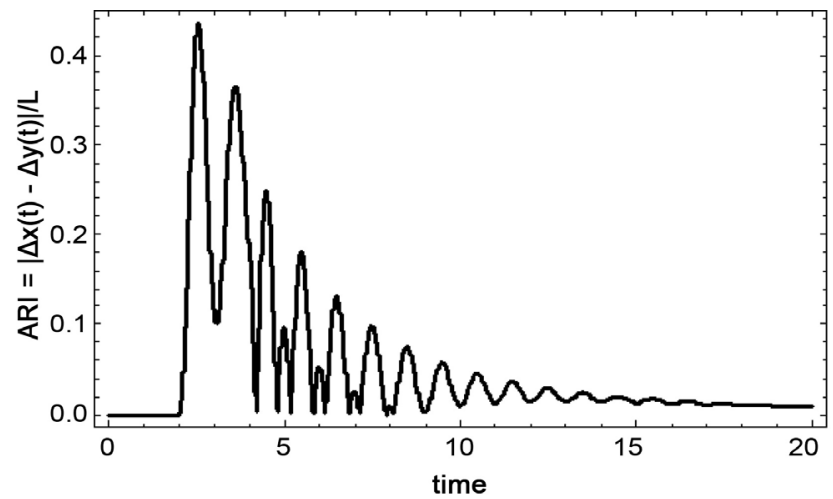

(b)

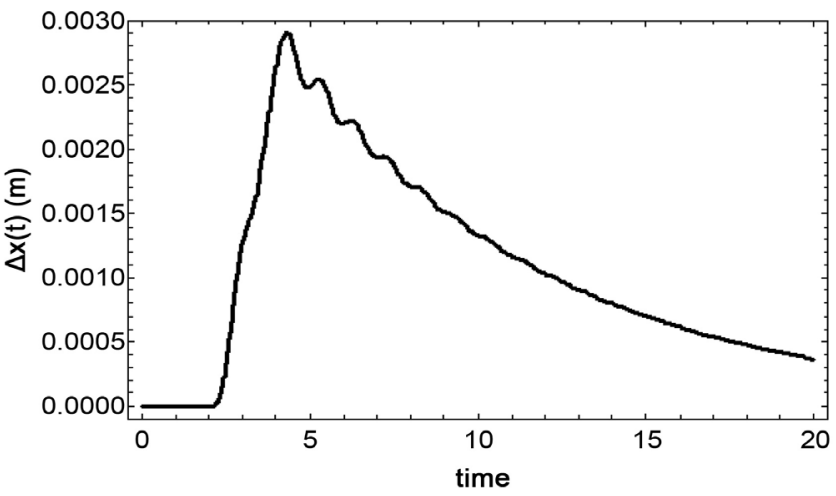

(d)

Figure 2. The one-convex rectangular impact, $z(t)$, and associated IRI, $\Delta \alpha(t)$ and $\Delta x(t)$. (a) The one-convex rectangular impact; (b) The associated roughness index (ARI) of one-convex rectangular impact; (c) The acceleration, $\Delta \alpha(t)$; (d) The variation of position, $\Delta x(t)=x(t)-x_{G}$ 
The numerical results of the associated roughness index (ARI), acceleration and position produced by the road-surface profile 2(a) are shown in Figures 2(b)-(d). The road-surface profile $z(t)$ in Figure 2(a) means a paved, smooth road surface with a roughness given by a rectangular impact (the height, $h_{1}=1.0(\mathrm{~cm})$, and time interval, $\left.\Delta t_{w}=\left|t_{1}-t_{\text {endl }}\right|=2 \quad(\mathrm{~s})\right)$. The abrupt longitudinal spikes in $\Delta \alpha(t), \Delta x(t)$ and ARI show the response to the impact. It should be noted that the smooth, damped oscillation corresponds to a smooth road surface. Hence, the abrupt longitudinal spikes and a group of smoothly damping spikes correspond respectively to a rough and flat road-surface. These are important properties for interpreting profiles of a roughness index.

The position $x(t)$ shows a smooth oscillation-damping from $\Delta x_{\max } \sim 0.30$ $(\mathrm{cm})$ to 0 , and the solution $y(t)$ shows a similar oscillation-damping from $\Delta y_{\max } \sim 0.40(\mathrm{~cm})$ to 0 . The maximum impact on $\Delta x_{\max }$ appears slightly delayed in time, which is a characteristic retardation phenomenon in coupled spring systems. The retardation effects of road-surface impacts are checked by comparing spikes between acceleration and position data in Figure 2. The impacts and retardation effects should be carefully examined when complicated, realistic road profiles are considered.

The simple patterns of motions in Figure 2 display one of basic properties of complicated real data which include unknown three-dimensional vibrations and trembles. The results of one-convex rectangular impact is a starting reference for scrutinizing the roughness of a road-surface. It is straightforward to represent a dent of road-surface by a concave impact, but because the IRI is originally defined as positive definite, it cannot distinguish the effects of convex or concave impacts on a road surface (the IRI could be defined without positive definite at the beginning). This will be shown in the following simulations.

\subsection{2. $z(t)=$ Four Convex-Concave Rectangular Impacts: Figure 3(a)}

The road-profile $z(t)$ with four convex-concave rectangular impacts with different impact strengths, $h_{i}(i=1,2,3,4)$ and solutions, $\Delta \alpha(t), \Delta x(t)$ and ARI, are shown in Figure 3.

The rectangular impacts are arbitrarily chosen to see patterns of acceleration, position and ARI. The maximum change of $|\Delta x(t)|$ is about $0.4(\mathrm{~cm})$ with respect to the impacts, $-1.0-2.0(\mathrm{~cm})$, and appears slightly delayed in an overall oscillation decrease. The ARI-data shows certain correlation between longitudinal spikes and convex-concave impacts. However, one can clearly notice that the IRI data cannot distinguish between convex and concave impacts because the IRI is positive definite.

The results indicate that a group of sharp longitudinal spikes corresponding to a rough road-surface and several smooth, damped oscillations represent flat road surfaces. It may be difficult for human perception to distinguish between smooth damped oscillations and irregular spikes when a road-surface becomes very rough. The complicated three-dimensional vibrations of a measuring system or 


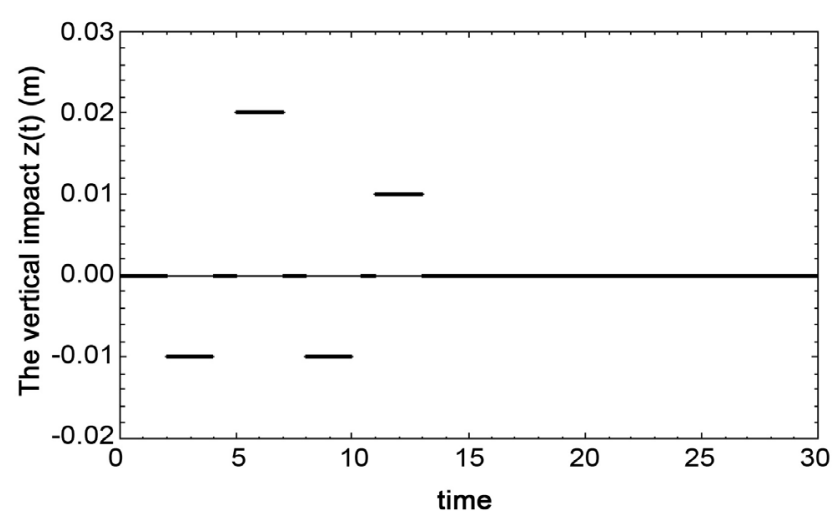

(a)

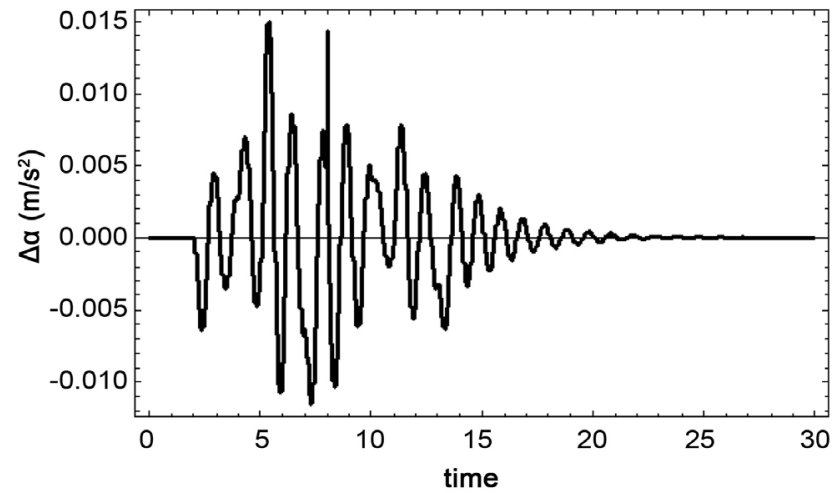

(c)

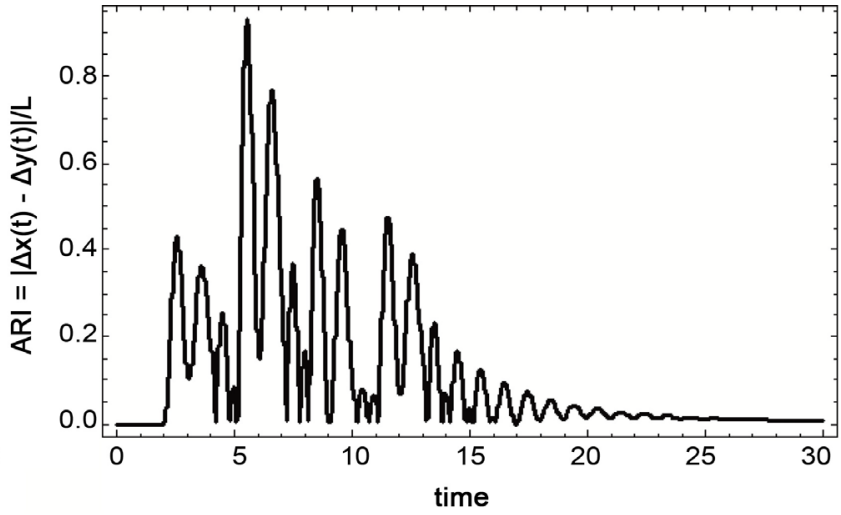

(b)

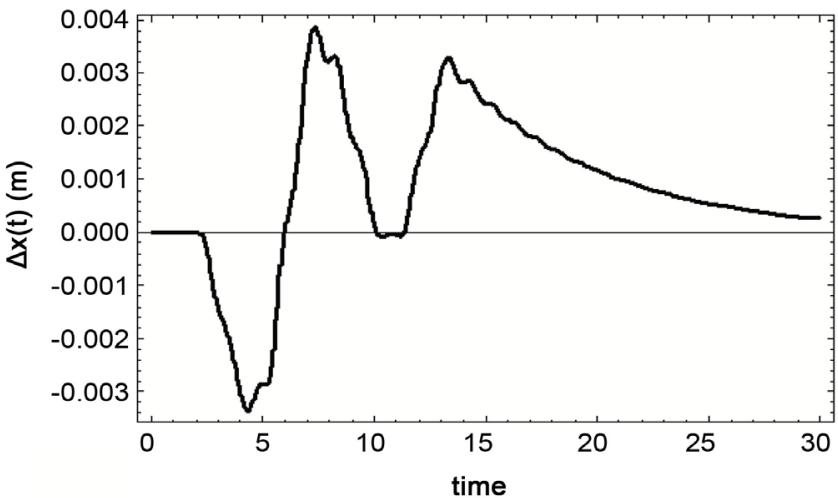

(d)

Figure 3. The four convex-concave impacts $z(t)$; the associated RI, $\Delta \alpha(t)$ and $\Delta x(t)$.(a) The four convex-concave rectangular impacts. (b) The associated roughness index (ARI) of four convex-concave rectangular impact. (c) The acceleration, $\Delta \alpha(t)$. (d) The variation of position, $\Delta x(t)=x(t)-x_{G}$.

Golden car could affect the measurement of data. However, the two-dimensional simulations could suggest that a computerized pattern-recognition may be possible by way of artificial intelligence (AI), if we supply sufficient patterns of vibrations and correlations between spikes and impacts.

The associated roughness index (ARI) seems to behave similarly as the IRI data, and so, the current two-dimensional, two-stratified spring model could support and compensate understanding profiles of IRI, which should be, in principle, checked by a three-dimensional model. The simulations of basic functions for road-surface, $z(t)$, and corresponding profiles for $\Delta \alpha(t)$, $\Delta x(t)$ and ARI will be continued.

\subsection{3. $z(t)=10$-Cluster Convex-Concave Rectangular Impacts + 10-Cluster Convex-Concave Rectangular Impacts: Figure 4(a)}

The 10-cluster +10 -cluster impacts in Figure 4 are shown for the simulation, and the associated roughness index (ARI), $\Delta \alpha(t)$ and $\Delta x(t)$ are shown. The strength of impacts, $h_{i}(i=1,2, \cdots, 20)$ are arbitrarily chosen in the current simulation. The sharp spikes in Figure 4(c) correspond to positive and negative impacts, and accumulations of impacts appear in a slightly time-delayed, retardation effect. However, the positive and negative impacts of a rough 


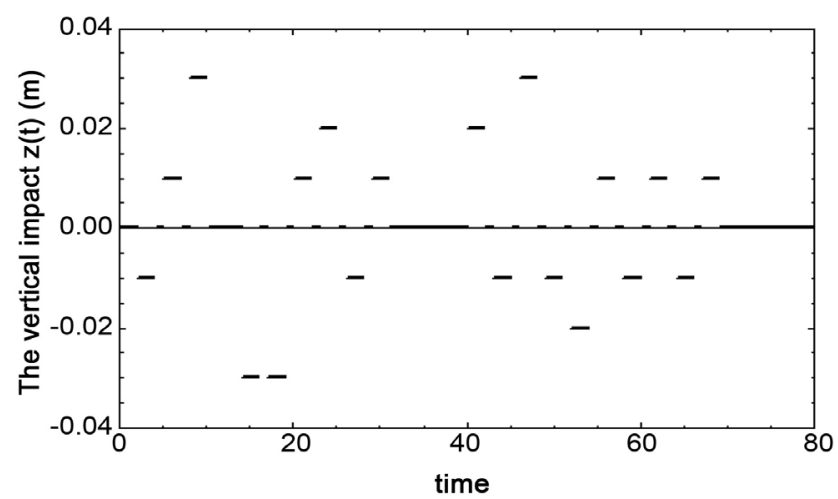

(a)

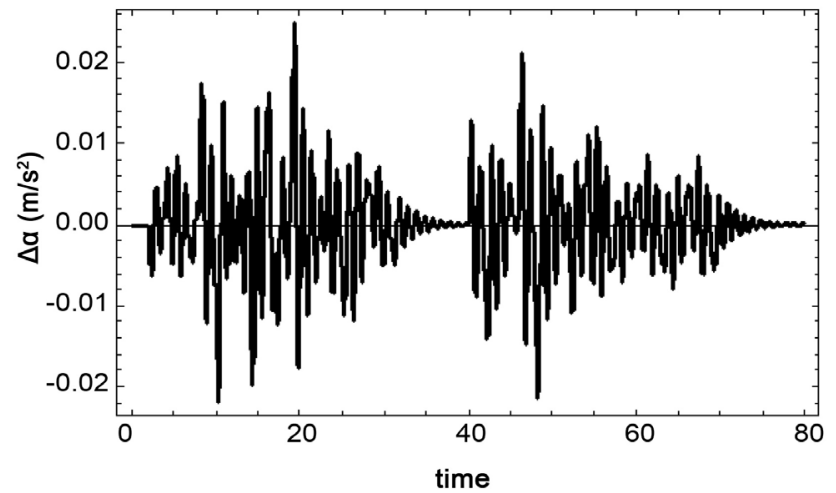

(c)

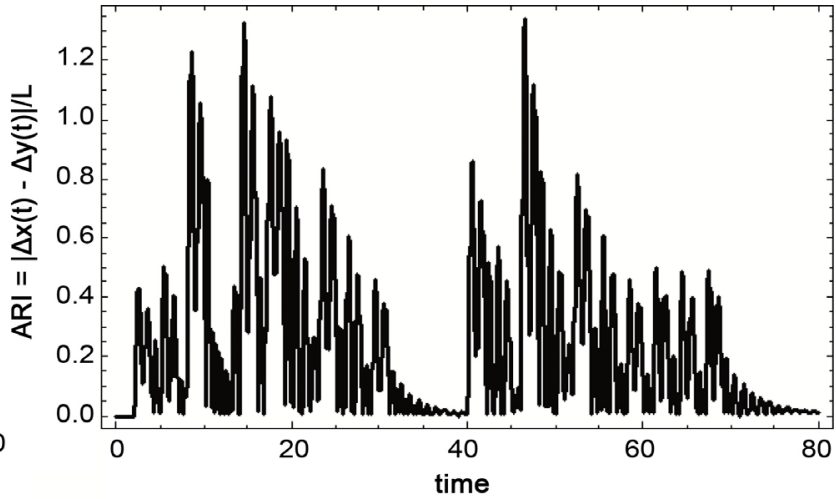

(b)

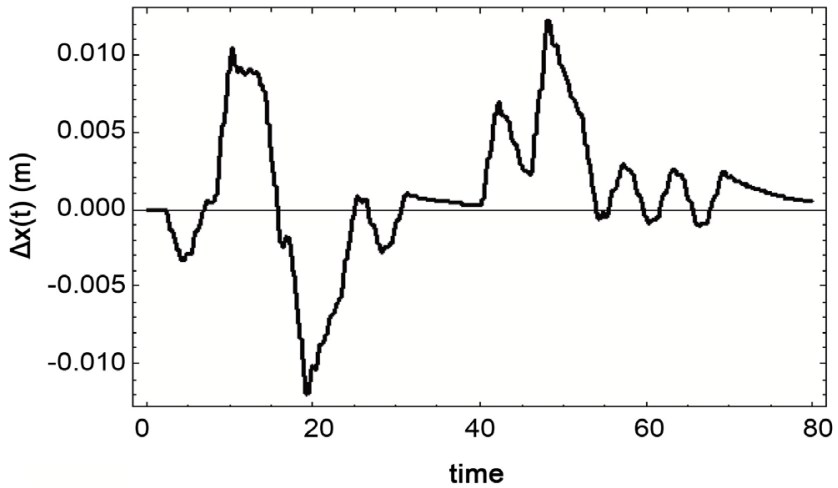

(d)

Figure 4. The profiles of $z(t): 10$ convex-concave +10 convex-concave impacts of 3 ; the associated RI, $\Delta \alpha(t)$ and $\Delta x(t)$. (a) The 10-cluster +10 -cluster convex-concave rectangular impacts. (b) The associated roughness index (ARI) of Figure 4(a). (c) The acceleration, $\Delta \alpha(t)$. (d) The variation of position, $\Delta x(t)=x(t)-x_{G}$.

road-surface $z(t)$ seem to be understandable with the signals of the ARI, $\Delta \alpha(t)$ and $\Delta x(t)$, which compensates the profile of IRI.

One can notice that the profiles of ARI, $\Delta \alpha(t)$ and $\Delta x(t)$ are gradually developing characteristics of IRI data. The smooth damped oscillations of $\Delta \alpha(t)$ in Figure $4(\mathrm{c})$ and $\Delta x(t)$ in Figure 4(d), in the time range $30(\mathrm{~s}) \lesssim t \lesssim 40(\mathrm{~s})$ and $70(\mathrm{~s}) \lesssim t \lesssim 80(\mathrm{~s})$, represent a smooth road surface. The sudden irregular spikes represent roughness of road-surface, and smooth damping spikes correspond to smooth road surface. Though they seem difficult to distinguish by human inspections, it may be possible to classify data for automated pattern recognition algorithm of artificial intelligence (AI). The automatic discovery of regularities through the use of computer algorithms may be applied to classify profiles among $z(t)$ and ARI, $\Delta \alpha(t)$ and $\Delta x(t)$.

The current simplified two-dimensional model can exhibit reasonable and realistic simulations of IRI data. Several characteristic patterns of ARI can be produced by changing strengths of impacts. In case that the same rectangular impact in Figure 2(a) is repeated in 20 times, a characteristic pattern in ARI, $\Delta \alpha(t), \Delta x(t)$ can be observed, which makes one readily recognize a rough road-surface. In addition, the repeated impacts expose accumulations of small uprise motions coming from each impact, but the accumulation is converging. 
The motion would remind one, for instance, the pattern of a rider and a horse in a horse-racing, in which a rider compensates accumulations of repeated impacts by counterbalancing horse's up-down motions. One can recognize the rider and horse motion by comparing simulations of $\Delta x(t)$ and $\Delta y(t)$ (centers of gravity of $m_{1}$ and $m_{2}$ ).

The accumulations of impact and counterbalancing motion to keep a stable continuous motion are an interesting and important phenomenon observed in the model simulations. The current accumulations of convex-concave impacts are, precisely speaking, accumulations of discrete impacts. Continuous impacts and those effects will be shown in the following simulations. The two-dimensional model of spring system show realistic motions and suggest that variations of ARI, $\Delta \alpha(t)$ and $\Delta x(t)$, could be useful to understand and construct profiles of IRI.

\subsection{4. $z(t)=$ Smooth Upward and Downward Slopes: (Figure 5(a))}

Now, we need to know profiles of roughness index corresponding to smooth upward and downward slopes and their representations in the ARI, $\Delta \alpha(t)$ and $\Delta x(t)$. The slopes are measured relative to the local reference frame, which are precisely defined as the horizontal and longitudinal line through the center of gravity in the current model.

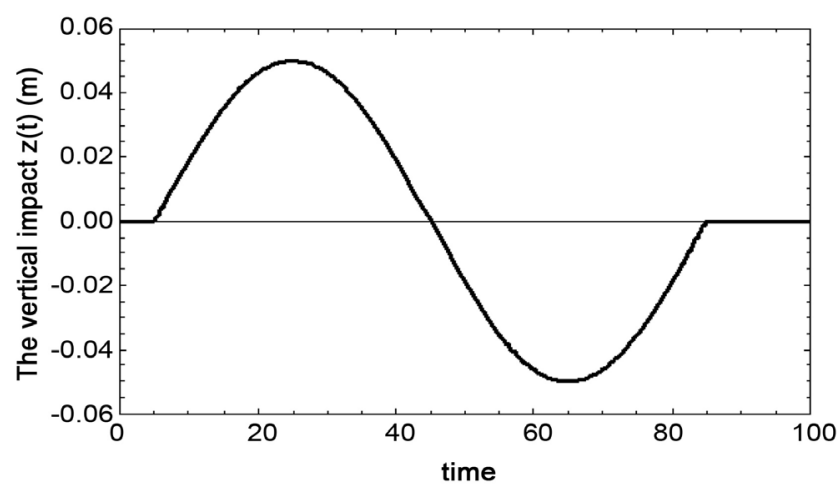

(a)

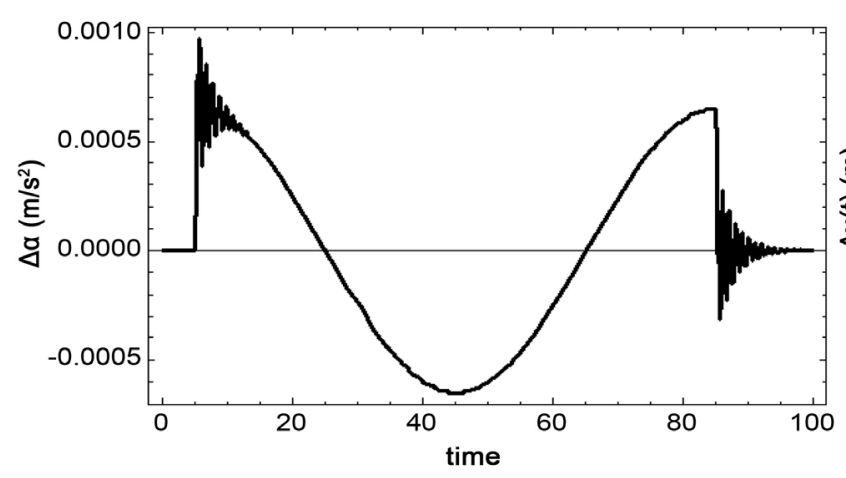

(c)

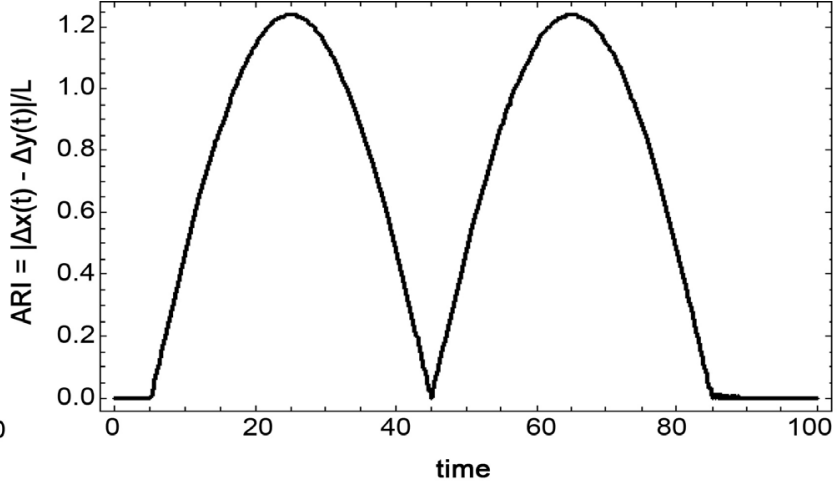

(b)

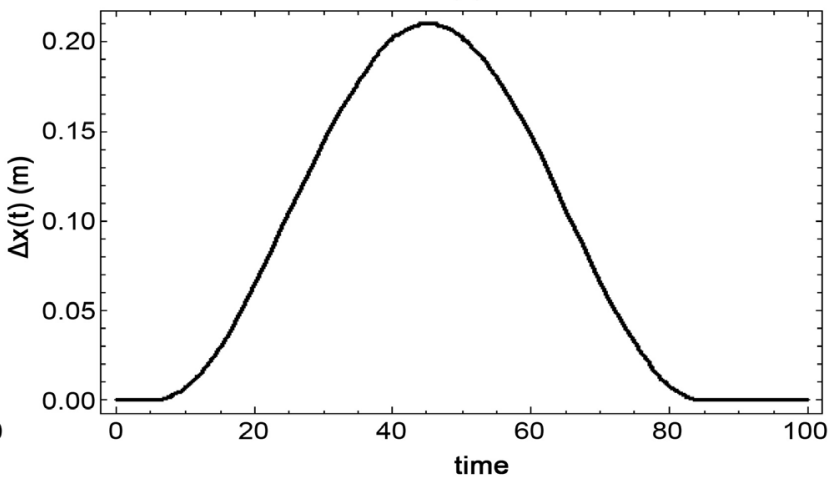

(d)

Figure 5. The profiles of $z(t)$ : Smooth upward and downward slopes of the impact of 4 ; the associated RI, $\Delta \alpha(t)$ and $\Delta x(t)$. (a) The smooth upward and downward slopes defined by $z(t)=A \sin (2 \pi t / T)$. (b) The associated roughness index (ARI) of Figure 5(a). (c) The acceleration, $\Delta \alpha(t)$. The variation of position, $\Delta x(t)=x(t)-x_{G}$. 
The smooth sinusoidal curve, $z(t)=A \sin (2 \pi t / T)$, is used to simulate continuous upward and downward slopes; $A$ and $T$ are the amplitude and period to characterize the slope, which are fixed as $A=5(\mathrm{~cm})$ and $T=80$ (s), respectively. This is a very smooth slope, and one should note that a Segway-type measuring system or a walking speed measurement is assumed in the present simulation. The functions $z(t)$ is reaching to the height $5(\mathrm{~cm})$ in $20(\mathrm{~s})$ and going down to $-5(\mathrm{~cm})$ in $40(\mathrm{~s})$, going back to a horizontal flat surface in $20(\mathrm{~s})$.

The simulation of sinusoidal $z(t)$ exhibits a smooth upward and downward curve in $\Delta x(t)$; the first upward curve of $\Delta x(t)$ corresponds to $T / 2$ of $z(t)$, and the last downward curve does to the next $T / 2$ of $z(t)$. It is noticeable that characteristic smooth damping oscillations at the beginning and ending are observed in $\Delta \alpha(t)$. The positivity of IRI exhibits only positive crests as shown in ARI contrary to a crest and a trough in $z(t)$.

The continuous accumulations of impacts from continuous sinusoidal $z(t)$ are observed by comparing Figure 5(a) and Figure 5(d). The amplitude of $z(t)$ is $A=5(\mathrm{~cm})$, but the impact force $-k_{2} z(t)$ exerting on the measuring system results in the crest about $20(\mathrm{~cm})$ in $\Delta x(t)$.

We suggest that IRI data should be reexamined by measuring the profile of smooth upward and downward road-surface data with accelerometer and GPS. It indicates that spikes on smooth upward and downward lines should be observed; the prediction is shown by the following simulation.

\subsection{5. $z(t)=$ Convex-Concave Impacts of Figure 5(a) with Smooth Upward and Downward Slopes (Figure 6(a))}

The upward and downward rough road surfaces are simulated by superposing both impacts of the convex-concave in Figure 4(a) and sinusoidal function in Figure 5(a). The discrete convex-concave impacts and continuous function $z(t)=A \sin (2 \pi t / T)$ are shown separately in Figure 6(a) for simplicity, but the superposition of both impacts is executed in numerical simulations.

The superposition of smooth slopes and convex-concave impacts exhibits a readily conceivable profile of roughness index as Figure 4(b) and Figure 6(b) are compared. The wide openings or empty spaces in Figure 6(b) are clearly observed and longitudinal spikes are dispersed in a curved line rather than a horizontal road-surface as in Figure 4(b). Collections of longitudinal spikes on a upward or a downward curves could be expected in IRI data, which corresponds to rough upward or downward slopes.

\section{Conclusions}

The computer simulations of IRI by the two-dimensional vehicle provided us with reasonable, realistic and new information by way of $z(t)$, ARI, $\Delta \alpha(t)$ and $\Delta x(t)$, though the two-dimensional model seemed a rough approximation for complicated three-dimensional system of vehicles. In addition, the assumption of flatness of a road-surface is not necessary for the center of gravity analyses. The appropriate measuring system and data comparable with the 


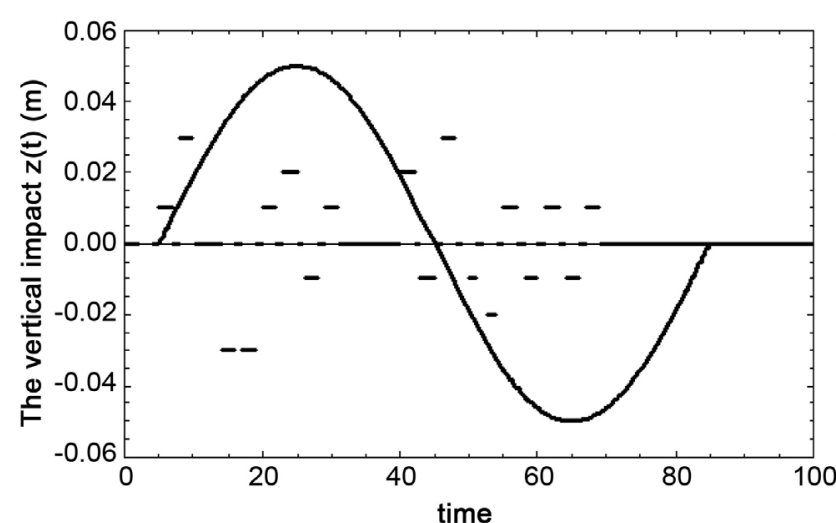

(a)

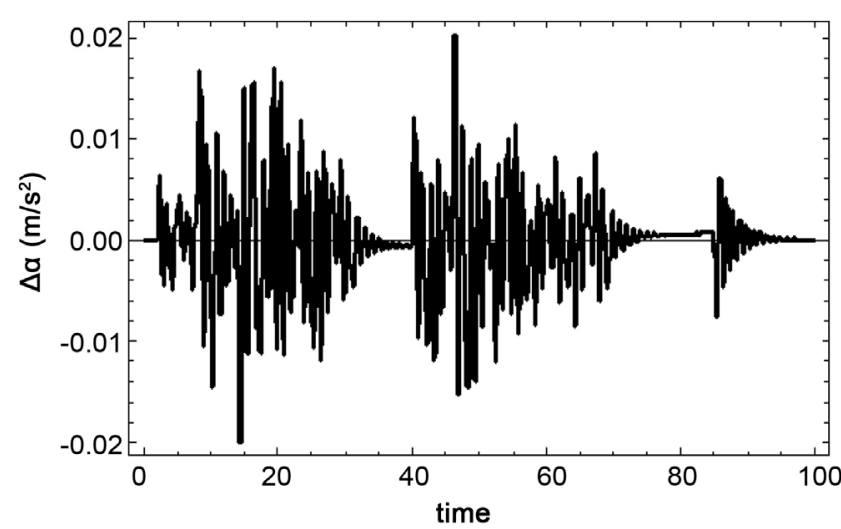

(c)

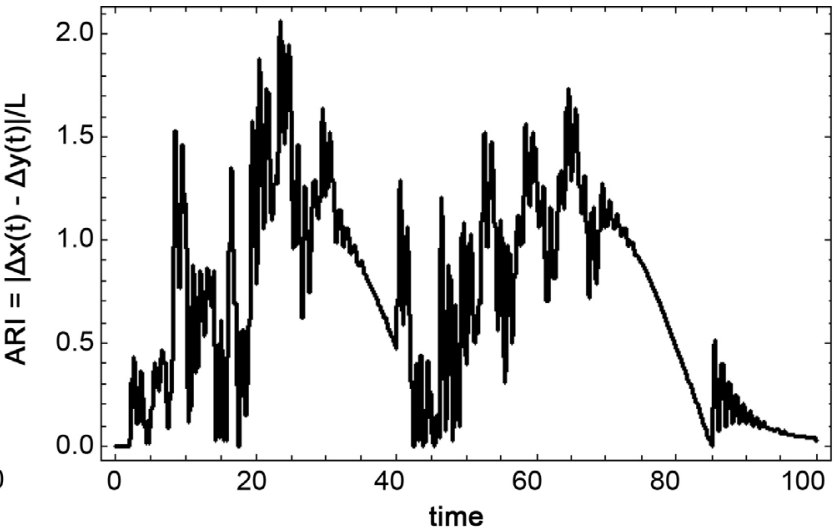

(b)

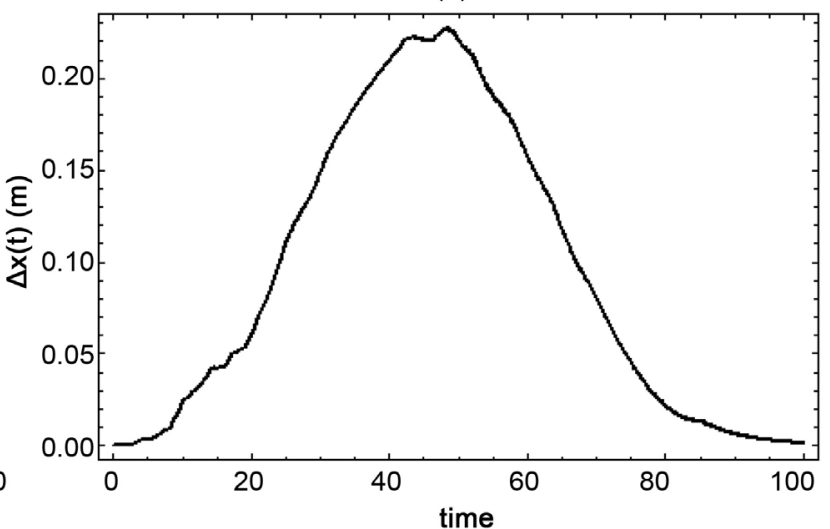

(d)

Figure 6. The profiles of $z(t)$ : convex-concave impacts of Figure 6(a) with smooth upward and downward slopes; the ARI, $\Delta \alpha(t)$ and $\Delta x(t)$. (a) The convex-concave impacts of Figure 4(a) with smooth upward and downward slopes. (b) The associated roughness index (ARI) of Figure 6(a). (c) The acceleration, $\Delta \alpha(t)$. (d) The variation of position, $\Delta x(t)=x(t)-x_{G}$.

two-dimensional model would be those measured by a walking speed or Segway: the two-wheeled, self-balancing personal transporter. The IRI data measured by Segway will be expected to have more reasonable correspondences with the roughness index supplied by two-dimensional model.

The two-dimensional, two-stratified spring system has exhibited reasonable, and realistic concepts, such as retardation phenomena, continuous and discrete accumulations of impacts, characteristic properties of road-surface impacts and profiles of road-surface roughness index. It would be appropriate to conclude that the coupled two-dimensional model provides us with data compatible with three-dimensional IRI, and the two-dimensional model simulations can provide helpful information for pattern-recognition, which could be better assisted by AI.

The coupled two-stratified spring system should be extended, for instance, to a three-stratified spring system with horizontal degrees of freedom. Though it would become much complicated computations, it could be possible with modern computers in order to examine three-dimensional modern vehicles of advanced suspension system and profiles of IRI data. The suspension designs in 
the current model are introduced with coil springs, but leaf springs and other sophisticated suspension schemes are commonly used in three-dimensional vehicles, such as tires, tire-air, and shock absorbers, providing a smooth, and comfortable ride-quality. Therefore, the current analysis is also related to the ride-quality of vehicles, minimization of damage and reduction of driver's fatigue on long journeys. The three-dimensional mechanical system is complicated vibrational system, but it is shown in the present paper that the two-dimensional model can explain characteristic vibrations for three-dimensional mechanical systems. The two-dimensional model and computer simulations will be helpful for studying mechanical vibrations as well as applications to ride-quality analyses of vehicles in general.

The profile of road-surface such as roughness, smoothness and curvature can be measurable by examining series of consecutive changes of acceleration, position and ARI data. The spacecraft rover or vehicle for exploring the surface of an extraterrestrial body (moon or Mars, etc.) could use and send information examined in the paper, because the theoretical analysis is effective in any gravity. Other possible, interesting applications of spring systems will be investigated in the future.

\section{Conflicts of Interest}

The authors declare no conflicts of interest regarding the publication of this paper.

\section{References}

[1] Gillespie, T.D., Sayers, M.W. and Segel, L. (1980) Calibration of Response-Type Road Roughness Measuring Systems. National Cooperative Highway Research Program.

[2] Spangler, E.B. and Kelly, W.J. (1964) GMR Road Profilometer, a Method for Measuring Road Profile, Research Publication GMR-452. General Motors Corp., Warren, $\mathrm{MI}$.

[3] Sayers, M.W., Gillespie, T.D. and Paterson, W.D. (1986) Guidelines for the Conduct and Calibration of Road Roughness Measurements. World Bank Technical Paper No. 46, The World Bank, Washington DC.

[4] The National Cooperative Highway Research Program (NCHRP) Conducts Research in Problems, Such as Highway Planning, Design, Construction, Operation, and Maintenance in the United States (Established in 1962). Federal Highway Administration and US Department of Transportation.

[5] Sayers, M.W. and Karamihas, M.S. (1998) The Little Book of Profiling. The Regent of the University of Michigan, Ann Arbor.

[6] Du, Y., Liu, C., Wu, D. and Jiang, S. (2014) Measurement of International Roughness Index by Using Z-Axis Accelerometers and GPS. Mathematical Problems in Engineering, 2014, Article ID: 928980.

[7] Kropac, O. and Mucka, P. (2009) Classification Scheme for Random Longitudinal Road Unevenness Considering Road Waviness and Vehicle Response. Shock and Vibration, 16, 273-289. https://doi.org/10.1155/2009/935858 
[8] McCuskey, S.W. (1962) An Introduction to Advanced Dynamics. Addison-Wesley, Boston.

[9] Wells, D.A. (1967) Lagrangian Dynamics. McGraw-Hill, New York.

[10] Logan, J.D. (1977) Invariant Variational Principles. Academic Press, Cambridge.

[11] Goldstein, H. (1980) Classical Mechanics. Addison-Wesley, Boston.

[12] Feynman, R., Gottlieb, M. and Leighton, R. (2013) Feynman's Tips on Physics: A Problem-Solving Supplement to the Feynman Lectures on Physics. Basic Books, New York.

[13] Sayers, M.W. (1995) On the Calculation of International Roughness Index from Longitudinal Road Profile. Transportation Research Record 1501.

[14] Sayers, M.W. and Karamihas, M.S. (1996) Interpretation of Road Roughness Profile Data. Federal Highway Administration Contract DTFH 61-92-C00143.

[15] Uechi, H. (1995) A Note on the Operator Method to Solutions of Linear Differential Equations. Bulletin of the College of Science, University of the Ryukyus, No. 59, 9-16.

[16] Koichi, Y. (2014) Collecting Pavement Big Data by Using Smartphone. IRF Asia Regional Congress Paper.

[17] The Australian Road Research Board (ARRB). https://www.arrb.com.au/about-arrb 